\title{
LAMIO AMPLEXICAULIS-URTICETUM URENTIS ASS. NOV. EN VIÑEDOS DE Mendoza, Argentina
}

\author{
EDUARDO MÉNDEZ1
}

\begin{abstract}
Resumen: Este estudio describe una nueva comunidad de malezas, la Lamio amplexicaulis-Urticetum urentis ass. nov. presente en viñedos consociados con olivos en Mendoza, Argentina. Para ello se utilizó el método fitosociológico de Braun-Blanquet relevando parcelas fisonómica, florística y ecológicamente homogéneas. La asociación es escíofila, basófila y nitrófila.
\end{abstract}

Palabras clave: Fitosociología, malezas, Lamio amplexicaulis-Urticetum urentis, Mendoza, Argentina.

Summary: Lamio amplexicaulis-Urticetum urentis ass. nov. in vineyards of Mendoza, Argentina. This study describes a new weed community, the Lamio amplexicaulis-Urticetum urentis ass. nov. present in vineyards consotiated with olive in Mendoza, Argentina. Using the Braun-Blanquet phytosociological method, a survey was conducted on physiognomically, floristically and ecologically homogeneous plots. The association is shade-bearing, basophilic and nitrophilous.

Key words: Phytosociology, weeds, Lamio amplexicaulis-Urticetum urentis, Mendoza, Argentina.

\section{INTRODUCCIÓN}

Durante estudios fitosociológicos de la vegetación arvense de los cultivos de la Provincia de Mendoza se encontró, en viñedos consociados con olivos, una comunidad de malezas dominada por Urtica urens L. Esta maleza de origen europeo ha sido registrada ampliamente en cultivos de Italia (Brullo \& Marcenó, 1983), España (Rivas Martínez, 1978, Ladero et al., 1981), Francia (Moutte, 1964), Alemania (Wilmanns, 1991), Chile (Matthei, 1995), Argentina (Marzocca et al., 1979, Ruiz Leal, 1973, Cerazo \& Conticello, 2008). En los estudios fitosociológicos de comunidades arvenses desarrollados en Mendoza (Méndez, 1983, 2002, 2003) no ha sido documentada la especie y tampoco como comunidad dominante.

El objetivo de este trabajo fue dar conocer través de su análisis fitosociológico, la comunidad de Urtica urens L.

\footnotetext{
${ }^{1}$ UID Botánica y Fitosociología IADIZA- CCT-CONICET Mendoza. Avda Dr. Adrián Ruiz Leal, $s / n^{\circ}$, Parque General San Martín, CP 5500, Mendoza, Argentina. El autor falleció en Marzo de 2015, estando en prensa este trabajo.
}

\section{Materiales y Métodos}

Esta comunidad se localiza en cultivos de vid (Vitis vinifera $\mathrm{L}$. var. Malbec) (contraespaldera o viña baja) consociada con olivo (Olea europaea L. var. Arauco) en el distrito Lunlunta, Dto. Maipú, en la provincia de Mendoza, Argentina, a aproximadamente $33^{\circ}$ $03^{\prime} 23,7^{\prime \prime} \mathrm{S}$ y $68^{\circ} 48^{\prime} 6,2^{\prime \prime} \mathrm{O}$ entre los 843 a $855 \mathrm{~m}$ s.m. El clima es cálido y seco (Norte 2000). De fina et al. (1964) registran para la localidad de Lunlunta temperaturas promedios anuales del mes más cálido de $23,1^{\circ} \mathrm{C}$ (enero) y del mes más frío (julio) de 5,7 ${ }^{\circ} \mathrm{C}$ y precipitaciones medias anuales de alrededor de $200 \mathrm{~mm}$, que la ubican en el distrito agroclimático Represa de las Viscacheras apto para los cultivos de vid y olivo. Los suelos son de origen aluvial y se corresponden con Torrifluvents típicos (INTA, 1990). Fitogeográficamente se la incluye en la provincia fitogeográfica del Monte (Cabrera, 1976).

La especie característica, guía y dominante de la comunidad es $U$. urens, planta anual, de tallos erguidos y flores en espigas.

La comunidad infestante se registró sólo en Lunlunta en dos fincas de 8 has total de cultivos y de un mismo establecimiento. La asociación se 
determinó por el método fitosociológico (BraunBlanquet, 1979) relevando stands de 10 a $20 \mathrm{~m} 2$. Todos los relevamientos (15) fueron realizados entre el segundo y tercer claro (distancia entre postes de la hilera) antes de los desagües y sobre superficies planas entre hileras (Foto 1). La información se completó con la del ciclo biológico, fenológico y origen (Marzocca et al., 1979, Campeglia, 1988), la formas de vida (Raunkiaer, 1905, 1937), y la presencia o grados de constancia (números romanos), obtenidos de los valores de abundancia-dominancia o cobertura de las especies (signo + y números arábigos) (BraunBlanquet, 1979). Se analizó la textura (método organoléptico), conductividad Eléctrica (CEA), fertilidad: Nitrógeno total (método Kjedahl), Fósforo por espectrometría (método Arizona) y Potasio por fotogrametría a la llama (método Pratt), materia orgánica por calcinación (método Davies) y pH con peachímetro de los suelos. Para ello se extrajeron, de los sitios de relevamientos, 5 muestras de suelos de los primeros $20 \mathrm{~cm}$ de profundidad las que fueron secadas al aire para su posterior análisis en laboratorio. Para conocer la variabilidad estadística de los análisis de suelos obtenidos se determinó la desviación estándar (DE) y coeficiente de variación (CV) a través de Infostat (Di Rienzo et al., 2011).

Para la identificación y nomenclatura de los taxones se utilizó a Marzocca et al. (1979), Ruiz Leal (1973), Zuloaga et al. (2008a, 2008b, 2008c). Para la nomenclatura de los taxones fitosociológicos se empleó a Barkmann et al. (1986) y Weber et al. (2000).

\section{Resultados}

Lamio amplexicaulis-Urticetum urentis ass. nov. (Tabla 1, Fig. 1). Holosyntypus: relev. 12.

\section{Ecología}

La asociación Lamio amplexicaulis-Urticetum urentis y la consociación de los cultivos de vid y olivo se desarrollan en suelos más o menos profundos, bien drenados de textura franco arenosa y con valores promedios de altos contenidos de materia orgánica $(\mathrm{MO}=3,75 \%, \mathrm{DE}: 0,11, \mathrm{CV}$ : 3,20), nitrógeno total $(\mathrm{N}=1419$ ppm, DE: 51,70, CV: 3,64), Fósforo $(\mathrm{P}=35,96 \mathrm{ppm}, \mathrm{DE}: 1,27$, CV: 3,54), Potasio ( $\mathrm{K}=482$ ppm, DE: 88,67, CV: $18,39)$, conductividad $(\mathrm{CEA}=3503,3 \mathrm{uS} / \mathrm{cm} 2$,
DE: $222,25 \mathrm{CV}: 6,34)$ y potencial hidrógeno $(\mathrm{pH}=$ 8,41, DE: 0,06, CV: 0,67). Por los bajos valores del coeficiente de variación, menores al $19 \%$, puede considerarse a los análisis de cada muestra de suelos como estadísticamente homogéneo. Cabe señalar que la mayoría de las especies dadas en la tabla 1 poseen valor indicador de Nitrógeno alto $(\mathrm{N}=8-9$, dentro de la escala de 0 a 9 de Ellenberg, 1974).

La asociación se comportaría como esciófila,basófila y nitrófila.

\section{Fisionomía}

El aspecto fisionómico más destacado lo da el color verde oscuro de las plantas de Urtica urens de 60 a $75 \mathrm{~cm}$ de alto. La comunidad es biestratificada y tiene cobertura total de 90 a $100 \%$ con una presencia de 7 a 11 especies (promedio 8,13).

A fines de invierno, mediados de septiembre, alcanza su óptimo florístico y de fructificación.

\section{Florística}

Las especies halladas en la asociación Lamio amplexicaulis-Urticetum urentis son de ciclo anual $(\mathrm{a}=81,0 \%)$ y perenne $(\mathrm{p}=19,0 \%)$. Domina la forma biológica terófíta $(\mathrm{T}=81,0 \%)$ sobre las geófitas $(\mathrm{G}=14,3 \%)$ y hemicriptófita $(\mathrm{H}=4,7 \%)$. Las especies anuales son de carácter invernal ( $\mathrm{I}=71,4$ $\%)$ y menores, de baja presencia, estivales ( $\mathrm{V}=$ $28,6 \%$ ). Igualmente la mayoría de las especies son introducidas $(\mathrm{i}=85,7 \%)$ señalando con ello la fuerte antropización de éstas áreas. La presencia de especies nativas en la composición florística $(\mathrm{n}=$ $14,3 \%$ ) le da matices propias a la comunidad.

Por su composición florística se la ubicaría sin inconvenientes en los taxones superiores de los esquemas sintaxonómicos de Brullo \& Marcenó (1983): Cl. Stellarietea mediae (Br.Bl. 31) R. Tx., Lohm. et Presg. in R. Tux. 1950 (Vegetación anual, a veces bianual, de sitios ricos en nitrógeno $(\mathrm{N}=$ 1419 ppm) y muy influenciado por el hombre: cultivos, terrenos abandonados). Ord. PolygonoChenopodietalia R. Tux et Lohm, in R. Tux. 1950 em. J. Tx. 1966 (vegetación inverno-primaveral, infestante de los cultivos, abonados, rico en nitrógeno). All. Lamio amplexicaulis-Urticion urentis all. nova prov. Ass. Lamio amplexicaulisUrticetum urentis ass. nov. (asociación anual invernal, nitrófila, basófila y de media sombra de cultivos consociados de vid con olivos). 
Tabla 1. Lamio amplexicaulis-Urticetum urentis ass. nov. en cultivos de vid (Vitis vinifera var. Malbec) con olivo (Olea europaea L. var. Arauco) de Mendoza.

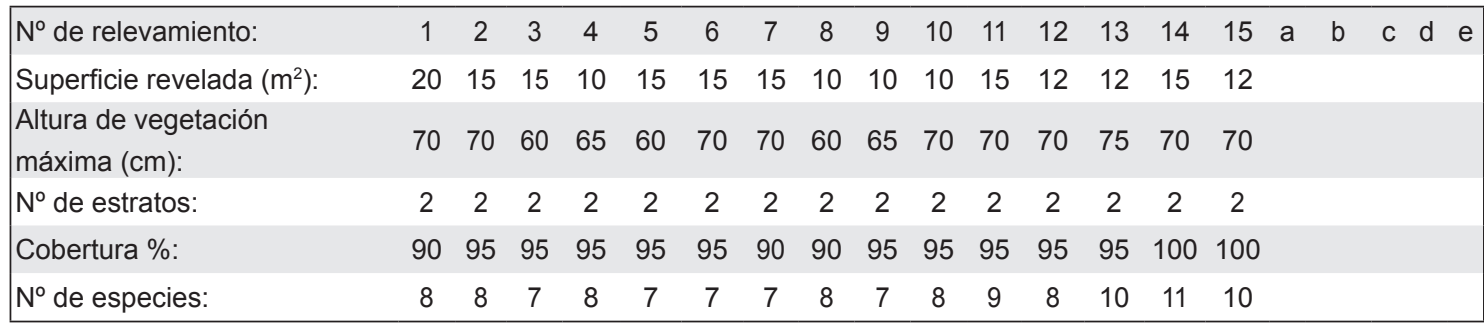

Ass. Lamio amplexicaulis -Urticetum urentis

Urtica urens L.

$\begin{array}{llllllllllllllllllll}4.4 & 4.4 & 5.4 & 5.4 & 5.4 & 5.4 & 4.4 & 4.4 & 5.4 & 5.4 & 5.4 & 5.4 & 5.5 & 5.5 & 5.4 & \text { a } & \text { T } & \text { I } & \text { V } & \text { i }\end{array}$

All., Ord. y Clase ( Lamio-Urticion, Polygono-Chenopodietalia y Stellarietea mediae)

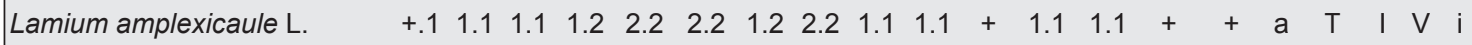

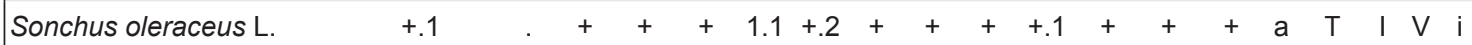

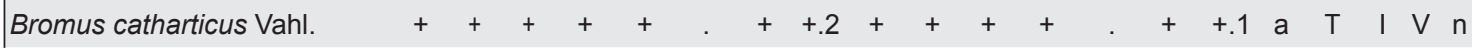
Malva parviflora L.

Poa annua L.

Fumaria parviflora Lam.

Stellaria media (L.) Vill.

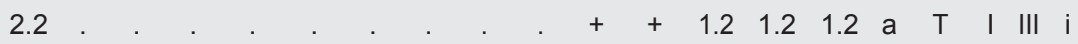

Coronopus didymus (L.) Sm.

Sisymbrium irio L.

Capsella bursa-

pastoris (L.) Medik.

Veronica persica Poir.

Senecio vulgaris $\mathrm{L}$.

Diplotaxis tenuifolia (L.) DC

Polygonum aviculare $\mathrm{L}$.

Chenopodium murale $\mathrm{L}$

Conyza bonariensis

(L.) Cronquist

Rumex crispus L.

Convolvulus arvensis L.

Wedelia glauca

(Ortega) Hoffmann

Cynodon dactylon (L.) Pers.

$\begin{array}{cccc}. & .2 & + & 1.2+. \\ + & + & + & . \\ 2.2 & . & . & .\end{array}$

\begin{tabular}{|c|c|c|c|c|c|c|c|c|c|c|c|}
\hline+ & . & . & + & 1.1 & + & + & + & a & $\mathrm{T}$ & I & IV \\
\hline & . & 1.2 & + & + & . & + & . & a & $\mathrm{T}$ & I & III \\
\hline & + & + & . & . & + & + & + & a & $\mathrm{T}$ & I & III \\
\hline$\cdot$ & . & . & + & + & 1.2 & 1.2 & 1.2 & a & $T$ & I & III \\
\hline T & +.1 & . & . & . & . & . & . & a & $\mathrm{T}$ & I & II \\
\hline & & & & & +1 & & + & a & $\mathrm{T}$ & | & \\
\hline
\end{tabular}

$+$

\begin{tabular}{|c|c|c|c|c|c|c|c|c|c|}
\hline . & . & . & + & . & + & $a$ & $\mathrm{~T}$ & 1 & I \\
\hline . & . & . & . & . & + & $a$ & $\mathrm{~T}$ & 1 & I \\
\hline . & . & . & . & + & . & $a$ & $\mathrm{~T}$ & I & I \\
\hline . & + & . & . & . & . & $a$ & $\mathrm{~T}$ & 1 & I \\
\hline . & . & . & . & . & . & $a$ & $\mathrm{~T}$ & 1 & I \\
\hline . & . & . & . & . & . & $a$ & $\mathrm{~T}$ & $\mathrm{~V}$ & I \\
\hline . & . & . & + & . & . & $a$ & $\mathrm{~T}$ & V & I \\
\hline+ & . & + & + & + & . & $p$ & $\mathrm{H}$ & $\mathrm{V}$ & III \\
\hline+ & . & . & . & . & . & $p$ & Griz & V & I \\
\hline . & . & . & . & . & . & $p$ & Griz & V & I \\
\hline & + & . & . & $r$ & . & $p$ & Griz & V & I \\
\hline
\end{tabular}

a: ciclo biológico: a: anual, p: perenne; b: Formas de vida: T: Terófita, H: Hemicriptófita, Griz: geófita rizomatosa; c: ciclo fenológico: I: Invernal, V: Estival, d: Presencia o Grados de Constancia: I (-20), II (2040), III (40-60), IV (60-80) y V (+80) (Braun-Blanquet, 1979). e: origen: i: introducida, n: nativa. Dentro de la tabla: valores de abundancia-dominancia o cobertura (signo + o números arábigos) de las especies de la comunidad: +(<1), 1(1-10), 2(10-25), 3(25-50), 4(50-75), 5(>75) (Braun-Blanquet, 1979). Todos los relevamientos en viñedos con olivos del Sr. Alonso, Lunlunta, Maipú,Mendoza, Argentina, a aprox. $33^{\circ} 03^{\prime}$ $23,7^{\prime \prime} S$ y $68^{\circ} 48^{\prime} 6,2^{\prime \prime}$ O, 843-855 m s.m., 17-18/9/2014. 
Bol. Soc. Argent. Bot. 50 (1) 2015

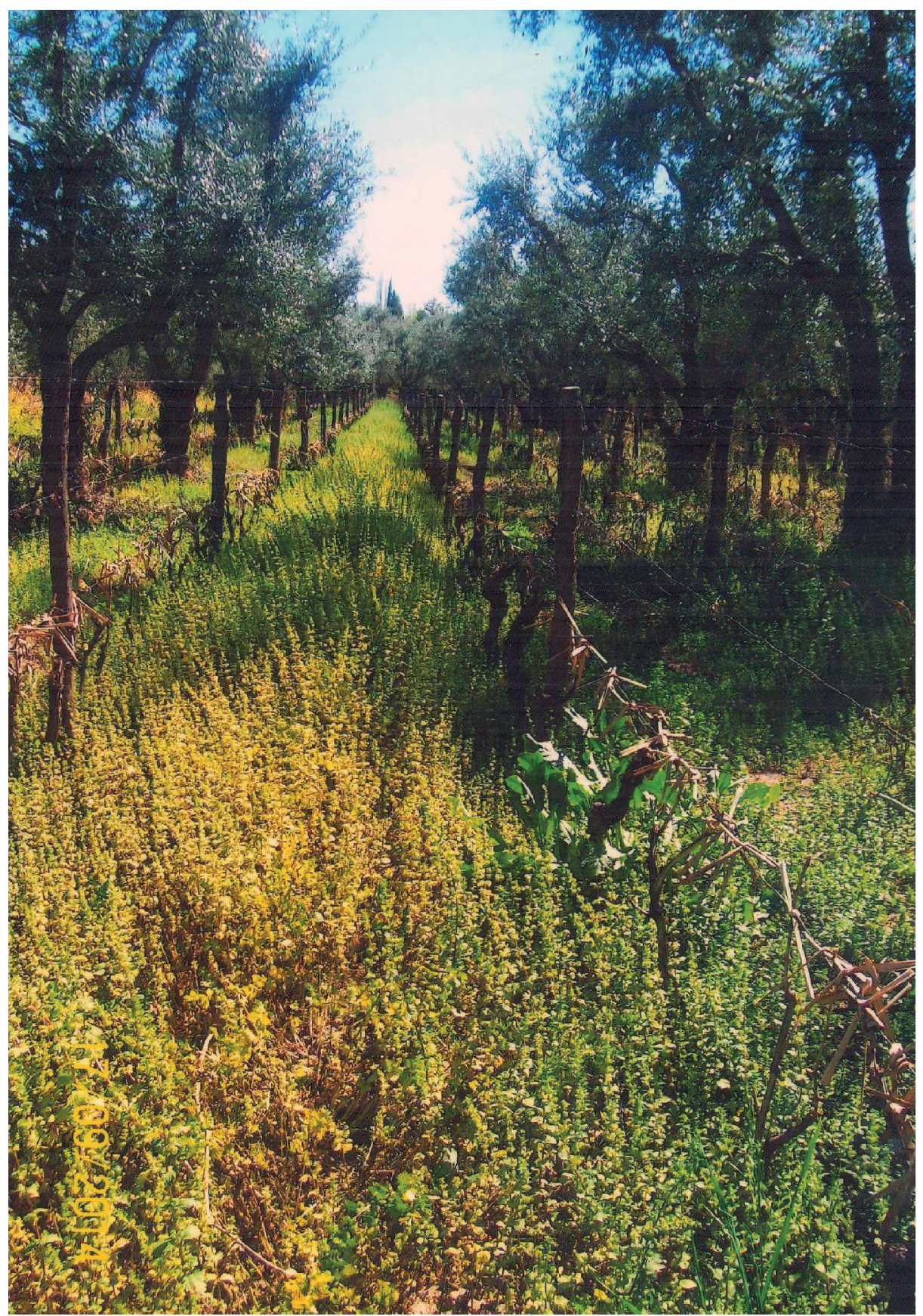

Fig. 1. Aspecto de la asociación Lamio amplexicaulis-Urticetum urentis en el viñedo con olivos.

\section{Discusión}

Enlabibliografíasintaxonómicase señalaaUrtica urens incluída en taxones europeos diferentes y hasta utilizando las mismas especies características para nuevas proposiciones sintaxonómicas. Así Brullo \& Marcenó (1983) la ubican formando parte del orden Urtico-Scrophularietalia peregrinae Brullo 1983, vegetación macrofítica anual esciófolo-nitrófila, la alianza Veronico-Urticion 
urentis Brullo1983 y las especies características Urtica urens y Veronica persica; Gehú et al. (1983) en su esquema sintaxonómico la ubican en el Orden Chenopodietalia muralis (Br. B1. 36 O. Bolós '62 emend. Rivas-Martínez 1978, vegetación ruderal anual muy nitrófila en el mediterráneo y norte de Europa dentro de la Allianza Chenopodion muralis Br.Bl. (31) 36 emend. O Bolós '96, con la asociación Chenopodio-Urticetum urentis Siss. 1966 en cultivos de suelos muy ricos en Nitrógeno; Izco et al. (2000) dan en el mismo Orden y Alianza anteriores la asociación Urtico urentisMalvetum neglectae (Knapp) 1945 Lohmeyer in Tux.1950; Ladero et al. (1981) también, en estas mismas unidades superiores, señalan la asociación Sisymbrio-Malvetum parviflorae RivasMartínez 1978 éstos últimos con las especies características Sisymbrium irio L., Urtica urens L, Chenopodium murale, Malva parviflora L. y otras especies comunes como, Stellaria media (L.) Will., Poa annua L, Lamium amplexicaule L., Senecio vulgaris L., Fumaria parviflora Lam., Veronica persica Poiret, Convolvulus arvensis L, entre otras. Esta asociación que describen, aún con bajos valores de coberturas de Urtica urens $(=1.10+.1)$, resulta ser la más afín a la nuestra.

Como se observa hay disparidad de criterios en la ubicación sintaxonómica de Urtica urens en diferentes asociaciones, alianzas, ordenes y clases. Esto nos lleva a proponer a la Lamio amplexicaulis-Urticetum urentis como una nueva asociación de malezas de los cultivos de viñedos consociados con olivos, por tener una identidad propia no solo por los elevados valores de coberturas (= 4 a 5 ) de Urtica urens sino desde un punto de vista ecológico definida por su comportamiento esciófila o de media sombra, en suelos muy ricos en Nitrógeno, materia orgánica y bases. Por otro lado la presencia de elementos nativos $(=14,3 \%)$ como Bromus catharticus Vahl., Conyza bonariensis (L.) Cronquist, Wedelia glauca (Ortega) Hoffmann la hacen diferente y válida propuesta para considerarla como una nueva asociación.

Con respecto a otras asociaciones de malezas descriptas para viñedos en la provincia de Mendoza, la Portulaco oleracea-Diplotaxietum erucoidis E.Méndez 2003 carece de Urtica urens, y tiene comportamiento estival, siendo fotófila, nitrófila, basófila $y$, aunque vive en suelos de textura semejantes, contiene valores más bajos de $\mathrm{N}=760$ ppm, $\mathrm{P}=6,05$ ppm, Mat. $\mathrm{Org}=0,87 \%$ y $\mathrm{pH}=7,18$, que los dados aquí para la Lamio amplexicaulisUrticetum urentis.

La composición florística de la Lamio amplexicaulis-Urticetum urentis, en cultivos de vid con olivos está asociada, además de las condiciones climáticas, a la intensidad de las labores culturales en el año pero sobre todo a las intensas y abundantes incorporaciones de materia orgánica. Mientras las condiciones climáticas definen la estacionalidad de las malezas invernales y estivales, las labores culturales contribuirían con el dinamismo de reemplazo de sus composiciones florísticas, en una situación análoga a los cambios florísticos estacionales de malezas en viñedos analizados en Mendoza (Méndez, en prensa). La instalación y mantenimiento de la Lamio amplexicaulisUrticetum urentis estaría condicionada a las labores culturales que favorecerían la alta presencia de terófitas $(\mathrm{T}=81,0 \%)$ y geófitas $(\mathrm{G}=14,3$ $\%$ ) en forma análoga a lo señalado en otras comunidades con Urtica urens como acompañante en el Diplotaxidetum erucoides Br.-Bl en viñedos de Mireval (Ocaña García, 1958), o de Francia (Moutte, 1964), y como dominante en cultivos hortícolas de Neuquén (Cerazo \& Conticello, 2008).

\section{Conclusión}

El estudio fitosociológico de esta asociación reveló la fuerte correspondencia con taxones europeos y particularmente con las malezas a niveles superiores de orden y clase como de los Polygono-Chenopodietalia y de los Stellarietea mediae, señalando el gran aporte de los mismos a otras regiones vitícolas como la nuestra con similaridad de manejos y cultivos. La asociación aquí descripta amerita ser considerada como tal no solo por sus propias características ecológicas, fisonómicas sino también florísticas a través de sus especies nativas.

\section{Agradecimientos}

A los revisores anónimos por sus correcciones y sugerencias del trabajo, a Enrique Mas por las 
determinaciones analíticas de los suelos, a Oscar Estévez por el análisis estadístico de los suelos, a Cecilia Sccones por el escaneo de la foto y al Sr. Alonso por permitir el ingreso a su finca.

\section{Bibliografía}

BARKMANN, J., J. MORAVEC \& S. RAUSCHERT. 1986. Code of phytosociological nomenclature. Vegetatio 32: 131-185. Den Hagg.

BRAUN-BLANQUET, J. 1979. Fitososociología. Bases para el estudio de las comunidades vegetales 1:819. Blume, Madrid.

BRULlO, S. \& C. MARCENÓ.1983. Contributo alla conoscenza della vegetazione nitrofila della Sicilia. Colloques phytosociologiques. Les vegetations nitrophiles et anthropogenes 12: 23-148. J. Cramer- Berlín.

CABRERA, A. L. 1976. Regiones Fitogeográficas Argentinas. Enciclopedia Argentina de Agricultura y Jardinería . 2 ed., 2 (1): 1-85. Ed. ACME, Buenos Aires.

CAMPEGLIA, O. G. 1988. Guía para el control de malezas con herbicidas en la provincia de Mendoza INTA Folleto 92: 5-9.

CERAZ0, M. B. \& L. CONTICELLO. 2008. Comunidades de malezas en cultivos hortícolas en la provincia de Neuquén (Argentina). Bol. Soc. Argent. Bot. 43(1-2): 121-135.

DE FINA, A. L., F. GIANNETTO, A. E. RICHARD \& L. SABELLA. 1964. Difusión geográfica de cultivos índices en la provincia de Mendoza y sus causas. INTA Inst. de Suelos y Agrotecnia, publ. no 83:1-38. Buenos Aires.

DI RIENZO, J. A., F. CASANOVES, M. G. BALZARINI, L. GONZÁLEZ, M. TABLADA \& C. M. ROBLEDO. 2011. InfoStat Version 2011. Grupo Infostat, FCA, Universidad Nacional de Córdoba. URL. http//wwwinfostat.com.ar.

ELLENBERG, H. 1974. Zeigerwerte der Gefabpflanzen Mitteuropas. Scrip. Geob.: 1-122.

GEHÚ, J. M., J. GEHÚ-FRANCK \& A. SCOPPOLA 1983. Schéma synsystématique des végétations nitrophiles et subnitrophiles de la région Nord/ Pas -De-Calais. Colloques phytosociologiques. Les Végétations nitrophiles et anthropogenes 12:567576. J. Cramer Berlín.

INTA. 1990. Atlas de suelos de la República Argentina. Provincia de Mendoza, Escala 1:1.000.000. Proyecto PNUD Arg 85: 71-106.

IZCO, J., J. AMIGO \& D. GARCÍA-LEÓN. 2000. Análisis y clasificación de la vegetación de Galicia (España), II. La vegetación herbácea. Lazaroa 21: 25.50 .
LADERO, M., O. SOCORRO, J. MOLERO MESA, M. LÓPEZ GUADALUPE, L. ZAFA, G. MARÍN, J. HURTADO \& F. R. ROYA. 1981. Algunas consideraciones sobre las comunidades nitrófilas de Granada (España). Actas III Congr. OPTIMA. Anales Jard. Bot. Madrid 37:737-763.

MATTHEI, O. 1995. Manual de malezas que crecen en Chile. Con la colaboración de C. Marticorena, R. Rodríguez y M. Quezada. Santiago de Chile.

MARZOCCA, A. O. S. MARSICO \& O. DEL PUERTO. 1979. Manual de malezas 1-564. Hemisferio Sur, Buenos Aires.

MÉNDEZ, E. 1983. Observaciones sobre la flora adventicia de viñedos en Mendoza. Parodiana 2: 263-276.

MÉNDEZ, E. 2002. Una comunidad de malezas en cultivos de Mendoza, Argentina: Brassisetum rapae ass. nov. Bol. Soc. Argent. Bot. 37: 93-37.

MÉNDEZ, E. 2003. Portulaco oleraceae-Diplotaxietum erucoides ass. nov. en viñedos de Mendoza, Argentina. Kurtziana 3: 41-44.

MÉNDEZ, E. En prensa. Cambios estacionales de las comunidades vegetales de malezas en viñedos de Mendoza, Argentina. Bol. Est. Geogr. UNCuyo.

MOUTTE, P. 1964. Etude phytosociologique et ecologique de la vegetation naturalle du vignoble d'Hyeres (var). Ann. Soc. Sc. Nat. Arch. Toulon: 82-106.

NORTE, F. 2000. Mapa climático de Mendoza. En: Argentina: Recursos y Problemas Ambientales de la Zona Árida 1: 25-27.

OCAÑA GARCÍA, M. 1958. Estudio fitosociológico de "La Guardiole" (Languedoc). Anal. del Inst. Bot. A. J. Cavanillies 16: 3-120.

RAUNKIAER, C. 1905. Typus biologiques pour la geographie botanique Bull. Acad. Sci. 5: 347-437.

RAUNKIAER, C. 1937. The life form of plants and Statistical plan geography.Claredon Press, Oxford.

RIVAS-MARTINEZ, S. 1978. Sobre la vegetación nitrófila del Chenopodion muralis Acta Bot. Malac. 4: 71-78.

RUIZ LEAL, A. 1973. Flora Popular Mendocina. Deserta 3: 3-273.

WEBER, J. E., J. MORAVEC \& J. THEURILLAT. 2000. International code of phytosociological nomenclatura -.3rd edición J. Veg. Sc. 11: 739-768.

WILMANNS, O. 1991. Phytosociology in vineyards: results, problems, tasks. In: Modern Ecology: basic and applied aspects. (Esser G. L. \& D. Overdiek (eds). Elsevier, Biomedical Press, Amsterdan, The Netherlands. London, New York, Tockie. Cap. 19: 399- 441.

ZULOAGA, F. O., O. MORRONE \& M. J. BELGRANO (eds.). 2008a. Catálogo de las Plantas Vasculares del Cono Sur (Argentina, Sur de Brasil, Chile, 
E. Méndez - Lamio amplexicaulis-Urticetum urentis ass. nov. en viñedos de Mendoza

Paraguay y Uruguay) Pteridophyta, Gymnospermae, Monocotyledoneae, Monogr. Syst. Bot. Missouri Bot. Gard. 107. Vol. 1: 1-983.

ZULOAGA, F. O., O. MORRONE \& M. J. BELGRANO (eds.). 2008b. Catálogo de las Plantas Vasculares del Cono Sur (Argentina, Sur de Brasil, Chile, Paraguay y Uruguay) Dicotyledoneae: AcanthaceaeFabaceae (Abarema-Schizolobium), Monogr. Syst. Bot. Missouri Bot. Gard. 107. Vol. 2: 985-2286.

ZULOAGA, F. O., O. MORRONE \& M. J. BELGRANO (eds.). 2008c. Catálogo de las Plantas Vasculares del
Cono Sur (Argentina, Sur de Brasil, Chile, Paraguay y Uruguay) Dicotyledoneae: Fabaceae (SennaZygia)-Zygophyllaceae, Monogr. Syst. Bot. Missouri Bot. Gard. 107. Vol. 3: 2287-3348.

Recibido el 2 de diciembre de 2014, aceptado el 2 de marzo de 2015. 
